\title{
METHOD OF ANALYSIS OF HIERARCHIES AND ITS APPLICATION TO THE PROBLEM "DEFINING PRIORITIES IN ASSESSING VARIOUS SKILLS AND COMPETENCES OF SOFTWARE ENGINEERS IN HIRING PROCESS"
}

\author{
Aghamehdi Mehdialiyev, Ph.D., Academy of Public Administration under the President, Baku, \\ Azerbaijan, \\ Seymur Farziyev, Founder of DevHq, Linz, Austria, \\ Samira Aliyeva, Senior Programmer at Azerconnect, Baku, Azerbaijan \\ DOI: https://doi.org/10.31435/rsglobal_conf/30032021/7480
}

Abstract. In the paper to solve the problem was used the AHP method, in which a decision-making problem decomposes into a system of hierarchies of objectives and alternatives. The implementation of the method of analysis of hierarchies on the defining priorities in assessing various skills and competencies of software engineers in hiring process is described.

Keywords: hierarchical structure, scale for relative importance, eigenvector, eigenvalue, priorities of performance metrics, priorities of topics, micro service, API.

\section{Brief description of the method of analysis of hierarchies.}

The hierarchy analysis method is a systematic procedure for presenting the elements of any problem in a hierarchical form. This method is based on this expert of the problem under study, decomposed into simple components by the decision-maker, followed by pairwise comparisons its elements. As a result, the relative degree (intensity) of the interaction of elements in the hierarchy can be expressed. The judgments will be expressed numerically later on. The hierarchical analysis method involves the procedure of synthesizing plural judgments, prioritizing criteria, and finding alternative solutions.

The solution to the problem is the process of setting priorities in stages. In the first stage, the most important elements of the problem are identified, and in the second stage, the best way to verify observations, tests, and element assessments is determined. The next step is to develop a method for applying the solution and assessing its quality. This procedure is repeated until a complete picture of the problem has been obtained and all characteristics necessary to arrive at a solution have been taken into account. Re-assessments are carried out until full confidence has been achieved. The process is carried out on a sequence of hierarchies. In this case, the results obtained in one of them are used to study the next sequence. The method of hierarchical analysis systematizes the process of solving such a multi-level problem.

Finally, if we consider that intuition and subjective evaluation are the main source material for an individual's full understanding of his creative potential, then the judgment about the superiority of one element over another and the intensity of these judgments can be used to express inner feelings and tendencies. Judgments broaden the scope of communication by enlarging clusters and elements in hierarchies on a particular issue.

Such an approach to the solution of the problem of choice stems from the natural characteristics of people, such as logical and creative thinking, the definition of events and the relationships between them. It should be noted that human beings have two characteristic features of analytical thinking: first, to observe and analyze; the second is to establish relations between them by defining the interrelationships between observations and to synthesize these relations into the general perception of the observed. All this gives an idea of the principle of identity and decomposition, the principles of discrimination of comparative judgments and synthesis.

The principle of identity and decomposition implies the structuring of the problem in the form of hierarchies and networks, which is the first stage in the application of the method of analysis of hierarchies. In its most elementary form, the hierarchy is built from the top (from a management point of view) to the lowest level, which consists of a list of alternatives through intermediate levels (criteria on which subsequent levels depend).

Once the problem is fully understood in the form of a hierarchical or network structure, the question becomes: how can the criteria be prioritized and evaluated according to those criteria so that the most important option can be identified? 
It is known that the main purpose of measuring physical quantities is to compare them with each other. Measuring two quantities means determining how many times one of them is in the other. For example, if two straight-line pieces in geometry are given, we can determine how many times the second piece is larger than the first by taking the smallest of them and placing them in sequence on the second piece. In this case, the length of the small piece actually acts as a measure of length. If the measurements of length are millimeters, centimeters, etc., The dimensions between these two pieces are understood to be the comparison between these two pieces using these units of measurement. If a unit of measurement for a physical quantity is given, then it is said, that there is a scale of measurement for that physical quantity. It is well known, that measurement scales differ for different physical quantities. Examples include length, weight, time, money, temperature, and so on. Special measuring devices have been invented to measure a variety of physical quantities. The dimensions of the corresponding quantities are determined with a certain accuracy by means of these devices.

But how can social, political, emotional, and other factors that cannot be measured physically be compared?

Assume that there is no scale to compare certain types of items according to any parameter. For example, suppose you need to compare two stones of different shapes, A and B, by weight, but there is no device to determine their weight. In this case, we can take one of the stones in our right hand and the other in our left hand to estimate their relative weights. We can accomplish this by lifting the stones one at a time so as not to manipulate the senses, even if one hand may be stronger than the other. Of course, by conducting such "experiments", we cannot say that stone A is $3 \mathrm{~kg}$ heavier than stone B, but we can judge that stone A is "slightly" heavier than stone B or "very heavy". Similarly, Factors that cannot be quantified and which are not understood can be compared in the same manner.

In the analysis of hierarchies, the elements of a problem are compared in pairs based on the influences they have on a shared characteristic. ("weights" or "intensity"). A relative significance scale was developed for subjective pairwise comparisons (Table 1) [9].

Table 1. Saaty's nine-point scale for relative importance

\begin{tabular}{|c|l|l|}
\hline Stage of Scale & \multicolumn{1}{|c|}{ Verbal Judgement } & \multicolumn{1}{c|}{ Characteristics } \\
\hline 1 & Equal Importance & Two activities contribute equally \\
\hline 3 & Moderate Importance & $\begin{array}{l}\text { Experience and judgement moderately favour one } \\
\text { activity over another }\end{array}$ \\
\hline 5 & Strong Importance & $\begin{array}{l}\text { Experience and judgement strongly favour one activity } \\
\text { over another }\end{array}$ \\
\hline 7 & Very Strong Importance & $\begin{array}{l}\text { An activity is strongly favoured and its dominance } \\
\text { demonstrated in practice }\end{array}$ \\
\hline 9 & Absolute Importance & $\begin{array}{l}\text { The evidence favouring one activity over another is of } \\
\text { the highest possible order of affirmation }\end{array}$ \\
\hline $2,4,6,8$ & $\begin{array}{l}\text { Intermediate values bet-ween } \\
\text { the two dja-cent judgements }\end{array}$ & When compromise is needed \\
\hline
\end{tabular}

With the help from the experts, the following form of the tables are filled with the hierarchy of all the levels (Figure 1):

\begin{tabular}{|c|c|c|c|c|}
\hline Top level & Factor 1 & Factor 2 & $\ldots \ldots \ldots \ldots . . .$. & Factor N \\
\hline Factor 1 & 1 & & & \\
\hline Factor 2 & & 1 & & \\
\hline & & & 1 & \\
\hline Factor N & & & & 1 \\
\hline
\end{tabular}

Fig. 1. Relative significance scale

Let's say that as a result of the expert estimation on the basis of the scale of relative importance are numbers: $\omega_{1}, \omega_{2}, \omega_{3}, \ldots \omega_{\mathrm{n}}$. We form from these numbers the following matrix:

If to designate elements of this matrix by $a_{i j}, i, j=1,2, \ldots n$. Then we will obtain:

$$
A=\left[\begin{array}{ccccc}
\varpi_{1} / \varpi_{1} & \varpi_{1} / \varpi_{2} & \varpi_{1} / \varpi_{3} & \ldots & \varpi_{1} / \varpi_{n} \\
\varpi_{2} / \varpi_{1} & \varpi_{2} / \varpi_{2} & \varpi_{2} / \varpi_{3} & \ldots & \varpi_{2} / \varpi_{n} \\
\varpi_{3} / \varpi_{1} & \varpi_{3} / \varpi_{2} & \varpi_{3} / \varpi_{3} & \ldots & \varpi_{3} / \varpi_{n} \\
\ldots & \ldots & \ldots & \ldots & \ldots \\
\varpi_{n} / \varpi_{1} & \varpi_{n} / \varpi_{2} & \varpi_{n} / \varpi_{3} & \ldots & \varpi_{n} / \varpi_{n}
\end{array}\right]
$$




$$
A=\left[\begin{array}{ccccc}
1 & a_{12} & a_{13} & \ldots & a_{1, n} \\
1 / a_{12} & 1 & a_{23} & \ldots & a_{2, n} \\
1 / a_{13} & 1 / a_{23} & 1 & \ldots & a_{3, n} \\
\ldots & \ldots & \ldots & \ldots & \ldots \\
1 / a_{1, n} & 1 / a_{2, n} & 1 / a_{3, n} & \ldots & 1
\end{array}\right]
$$

The table on the left column of the elements of the first line in the elements, according to the relative importance of the appointment are. In other words, the left element in the above-mentioned elements is important, the table with the box, the relative importance scale shown from 1 to 9 as numbers one, the case of the numbers upside down, prices are recorded. Any element of self-according to the relative importance to the 1 equivalent so that the table diagonal elements, only 1 from a must. Table of other symmetrical fields, the one opposite the price is filled, the A element, B element "slight advantage" accepted are, then the table with the row and column intersection awarded scale of 3, the price is written and $\mathrm{B}$ element, the element according to the relative importance of this issue, reverse the price, $1 / 3$ of the characterization is. Table all the fields of the rule, with a filling the hierarchy of all levels down security $\mathrm{n}$ skew symmetric matrices [8].

But how objectively do the tables filled by the experts reflect the situation, or to what extent do the experts' opinions agree with each other? In general, the eigenvalues and eigenvectors of matrices compiled based on expert estimations for the correct levels of the hierarchical structure must be found by solving the following mathematical problem:

$$
A x=\lambda x \text {, }
$$

Where, $\lambda$ is a eigenvalue, and $\left.x=\left(x_{1}, x_{2}, x_{3}, \ldots x_{n}\right)\right)$ is a eigenvector.

It is known that for a reciprocal matrix takes place

$$
\lambda_{\text {max }} \geq n,
$$

Where, $\lambda_{\max }$ - greatest eigenvalue, $n$ - order of the matrix $A$. The equality sign takes place only for coherence matrices [11].

As noted above, for elements of each level of the hierarchical structure, the coefficients of relative importance are found as a solution of the eigenvalues and eigenvectors of the matrices of pairwise congruences. In the general case, there are strict mathematical methods for solving this problem. But, T. Saati in his book [8] proposed simple formulas for calculating eigenvalues and eigenvectors. These formulas are proposed in Figure 2.

$$
\begin{aligned}
& \mathrm{X}_{1}=\left(1^{*}\left(\mathrm{~W}_{1} / \mathrm{W}_{2}\right)^{*} \ldots{ }^{*}\left(\mathrm{~W}_{1} / \mathrm{W}_{\mathrm{n}}\right)\right)^{1 / \mathrm{n}} \\
& X_{n}=\left(\left(W_{n} / W_{1}\right)^{*} \ldots *\left(W_{n} / W_{n-1}\right)^{*} 1\right)^{1 / n} \\
& \lambda\left(A_{1}\right)=X_{1} / \Sigma X_{i} \\
& \lambda\left(A_{n}\right)=X_{n} / \Sigma X_{i}
\end{aligned}
$$

Fig.2. Formulas for calculating eigenvalues and eigenvectors.

Further, for each level, the consistency index (CI) is determined by the formula:

$$
C I=\frac{\lambda_{\max }-n}{n-1}
$$

After computing the CI from values scale $1 / 9,1 / 8,1 / 7, \ldots 1,2,3, \ldots, 9$ randomly formed the coherence matrices and for different orders are calculated random index (RI). Middle RI matrices for matrices of order from 1 to 10 , on the basis of 100 random samples are presented in the form of the following standard table [6].

Table 2. Average random number index for each size of the matrix

\begin{tabular}{|l|l|l|l|l|l|l|l|l|l|l|}
\hline $\mathrm{n}$ & $\mathbf{1}$ & $\mathbf{2}$ & $\mathbf{3}$ & $\mathbf{4}$ & $\mathbf{5}$ & $\mathbf{6}$ & $\mathbf{7}$ & $\mathbf{8}$ & $\mathbf{9}$ & $\mathbf{1 0}$ \\
\hline $\mathrm{RI}$ & $\mathbf{0}$ & $\mathbf{0}$ & $\mathbf{0 , 5 8}$ & $\mathbf{0 , 9}$ & $\mathbf{1 , 1 2}$ & $\mathbf{1 , 2 4}$ & $\mathbf{1 , 3 2}$ & $\mathbf{1 , 4 1}$ & $\mathbf{1 , 4 5}$ & $\mathbf{1 , 4 9}$ \\
\hline
\end{tabular}


In this table, the first line specifies the matrix size -n and on the second line the average RI.

Dividing, CI to RI receive ration consistency (RC).

$$
R C=\frac{C I}{R I}
$$

It is generally considered that for harmonised data RC must not exceed $0,1(10 \%)$, in some cases, $0,2(20 \%)$.

The expert analysis by the hierarchy analysis method is completed in two stages. In the first stage, the goals of the actors that best suit the overall purpose of the system are identified, and the most important ones are selected. In the second stage of expert assessment, the different scenarios identified through the hierarchical structure of the problem are first compared in pairs according to the "Relative Importance Scale" for each of these objectives. Then, in the same way as described above, the weights of the scenarios are determined and the attributes and characteristics of the municipal experts on the basis of the following table [8]:

Table 3. Scale of difference for comparison

\begin{tabular}{|c|l|}
\hline Difference in values & \multicolumn{1}{|c|}{ Definition } \\
\hline 0 & Value does not change \\
\hline $2(-2)$ & A small increase (decrease) in value \\
\hline $4(-4)$ & A large increase (decrease) in value \\
\hline $6(-6)$ & A significant increase (decrease) in value \\
\hline $8(-8)$ & The maximum increase (decrease) in value \\
\hline $1,3,5,7,-1,-3,-5,-7$ & Intermediate values between the two judgments \\
\hline
\end{tabular}

The calculated weights of the scenarios are multiplied by the appropriate estimates given to them by the experts, and on the basis of these estimates, the attributes and characteristics of the municipalities are assessed on an already generalized scale. Based on the values of the generalized scale, it is not difficult for the decision-maker to construct a generalized scenario.

In order to conclude the study, another question is necessary: how much can the solution be considered optimal? To answer this question, T. Saaty first introduced the concept of the "compromise index".

$$
\text { compromise index }=\frac{\lambda_{\max }-n}{n-1}
$$

Finally, the concept of the so-called "reconciliation ratio", which consists of the ratio of the coherence index to the random coefficient of coherence, is introduced ([1]):

$$
\text { reconciliation ratio }=\frac{\text { reconciliation index }}{\text { random reconciliation factor }} * 100 \%
$$

$\mathrm{T}$. Saaty reconciliation ratio obtained to solve the problem in hours

$$
\text { reconciliation ratio } \leq 10 \%
$$

If the condition is met, the solution of the issue is considered acceptable. If this condition is not met, the issue is reconsidered, the members of the expert group are asked to reconsider their assessments. Thus, the method of "analysis of hierarchies" allows you to search for a solution to the problem until a reasonable solution.

Application to the problem: defining priorities in assessing various skills and competencies of software engineers in hiring process.

Problem statement. DevHQ is the international company headquartered in Austria that helps their customers to assess technical qualification and performance of developer candidates in an automated and efficient way. (DevHq Web Page: https://devhq.de/)

The problem DevHQ solves leveraging AHP is as following: there should be developed a software solution which could assess technical performance and qualifications of candidates transparently without involving any human into the decision-making process. There is a list of certain technical metrics which is used in assessment. These metrics have certain priorities over others. DevHQ had the problem to transparently and correctly identify which metric has to be more prioritized over other metrics and how the priorities should be valuated with numbers.

Application of AHP. Below are some of the technical metrics and their weights (Figure 3): 


\begin{tabular}{|c|c|c|c|c|c|c|c|c|c|c|c|c|c|c|c|}
\hline SSD & LNE & WORD & SIIE: & LOGIC & UNT_TEST_COVERGGE & 106 & COOE COWEETTON & CODE $D O C$ & CODE COMUEST & GT_CONMT & GI PUSH & AIEN CODE & ECOEE SUELL & SECORTYY YULNERABUTT & 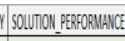 \\
\hline 1 & 7 & 7 & 7 & 0.14857113 & 0.25 & 5 & 1 & 5 & 9 & 7 & 3 & 1 & 2 & 0.111111111 & 0.2 \\
\hline 0.142857113 & 1 & 5 & 1 & 0.128871143 & 0.2 & 3 & 1 & 7 & 5 & 5 & 8 & 0.142857113 & 0.142857414 & 0.111111111 & 0.125 \\
\hline 0.142857113 & 02 & 1 & 1 & 0.1111111111 & 0.125 & 0.142857143 & 0.1111111111 & 0.2 & 0.2 & 9 & 5 & 9 & 9 & 9 & 9 \\
\hline 0.142857113 & 1 & 1 & 1 & 0.1111111111 & 0.142857143 & 0.2 & 0.125 & 033333333 & 0.2 & 0.1111111111 & 0.2 & 0.11111111111 & 0.142857143 & 0.111111111 & 0.111111111 \\
\hline 7 & 7 & 9 & 9 & 1 & 4 & 8 & 7 & 8 & 9 & 6 & 9 & 3 & 5 & 1 & 3 \\
\hline 4 & 5 & 8 & 7 & 0.25 & 1 & 8 & 5 & 8 & 8 & 6 & 9 & 3 & 3 & 0.1666666667 & 0.25 \\
\hline 0.2 & 0.333333333 & 7 & 5 & 0.125 & 0.125 & 1 & 0.2 & 1 & 1 & 0.1122577143 & 5 & 0.14285713 & 0.333333333 & 0.142857143 & 0.142857143 \\
\hline 1 & 1 & 9 & 8 & 0.12857113 & 0.2 & 5 & 1 & 9 & 8 & 5 & 9 & 0.5 & 5 & 0.142857143 & 0.125 \\
\hline 0.2 & 0.14285713 & 5 & 3 & 0.125 & 0.125 & 1 & 0.1111111111 & 1 & 1 & 0.142857143 & 1 & 0.14285713 & 0.2 & 0.111111111 & 0.1111111111 \\
\hline 0.1111111111 & 0.2 & 5 & 5 & 0.1111111111 & 0.125 & 1 & 0.125 & 1 & 1 & 0.142857143 & 1 & 0.14285713 & 0.2 & 0.111111111 & 0.111111111 \\
\hline 0.14287113 & 0.2 & 0.1111111111 & 9 & 0.166666607 & 0.1666666607 & 7 & 02 & 7 & 7 & 1 & 7 & 0.166666667 & 0.2 & 0.142857143 & 0.142857143 \\
\hline 0.333333333 & 0.125 & 0.2 & 5 & 0.1111111111 & 0.1111111111 & 0.2 & 0.1111111111 & 1 & 1 & 0.142857143 & 1 & 0.11111111111 & 0.142857143 & 0.111111111 & 0.1111111111 \\
\hline 1 & 7 & 0.11111111111 & 9 & 0.33333333 & 0.3333333333 & 7 & 2 & 7 & 7 & 6 & 9 & 1 & 5 & 0.2 & 0.1666666667 \\
\hline 0.5 & 7 & 0.1111111111 & 7 & 02 & 0333333333 & 3 & 0.2 & 5 & 5 & 5 & 7 & 0.2 & 1 & 0.1666666667 & 0.166666667 \\
\hline 9 & 9 & 0.11111111111 & 9 & 1 & 6 & 7 & 7 & 9 & 9 & 7 & 9 & 5 & 6 & 1 & 1 \\
\hline 5 & 8 & 0.111111111111 & 9 & 0.33333333 & 4 & 7 & 8 & 9 & 9 & 7 & 9 & 6 & 6 & 1 & 1 \\
\hline
\end{tabular}

Fig. 3. The technical metrics and their weights

From this matrix following values are calculated:

\section{Unnormalized weights}

The weight $w$ of each metrics $i$ of the unnormalized AHP matrix is calculated through the function below:

$$
\mathrm{w}_{\mathrm{i}}=\prod_{i=1}^{n}\left(C_{i j}\right)^{\frac{1}{n}}
$$

Here, $C_{i j}$ is the significance value of each metric $i$ in the row against the metric $j$ in the respective column. $n$ is the size of the metrics matrix. In our case above $n=16$

\section{Normalized priorities.}

Once weights have been calculated, unnormalized matrix should be normalized further with the following function to get normalized priorities for each metrics:

$$
p_{i}=\frac{w_{i}}{\sum_{i}^{n} w_{i}}
$$

For final assessment of the solution of the user, normalized priority of each metric is multiplied with its respective function of score and each product of multiplication is summed up:

$$
\Phi=\sum_{\mathrm{i}=1}^{\mathrm{n}}\left(\mathrm{p}_{\mathrm{i}} * \mathrm{~F}_{\mathrm{i}}\right)
$$

Here $F_{i}$ is a score function that calculates value for each metric $i$. Once the metric score has been calculated, its final score is calculated with the help of the gained AHP priority. The overall assessment result $\Phi$ is calculated for the candidate by summing up candidate's final metric scores which is in our example 16 different final metric scores with their corresponding priorities.

Whenever priorities of technical metrics gets updated in the given matrix above, assessment decision gets updated automatically. With this way DevHQ was able to provide solution to its customers to define their own decision making system flexibly and transparently.

The solution for this problem implemented as a standalone REST micro service and available through the following API documentations:

- https://lb.devhq.in/ahp/swagger-ui.html

- https://lb.devhq.in/ahp/monitoring/info

Some outputs from system.

As the usage of this system is secured from outside, some screenshots are shared as a reference (Figure 4, Figure 5.): 


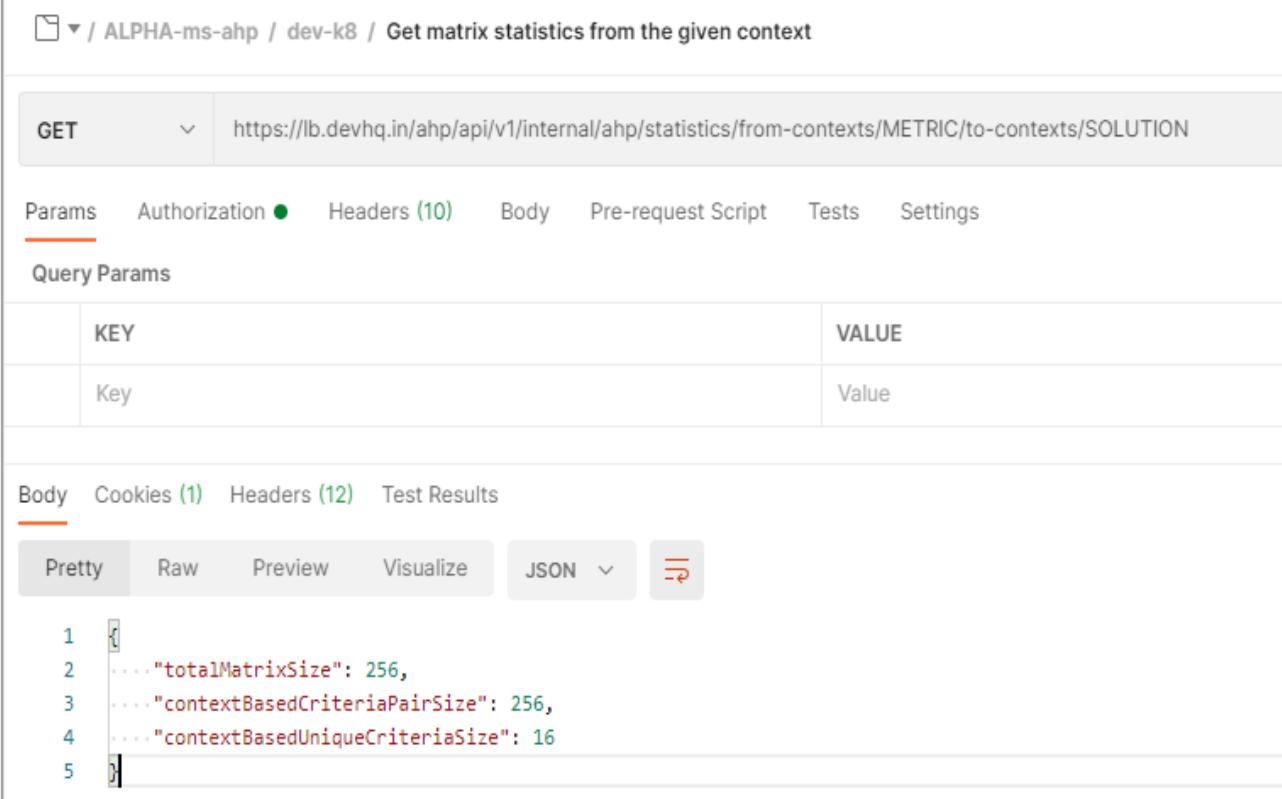

Fig. 4. Getting the statistics via AHP REST API

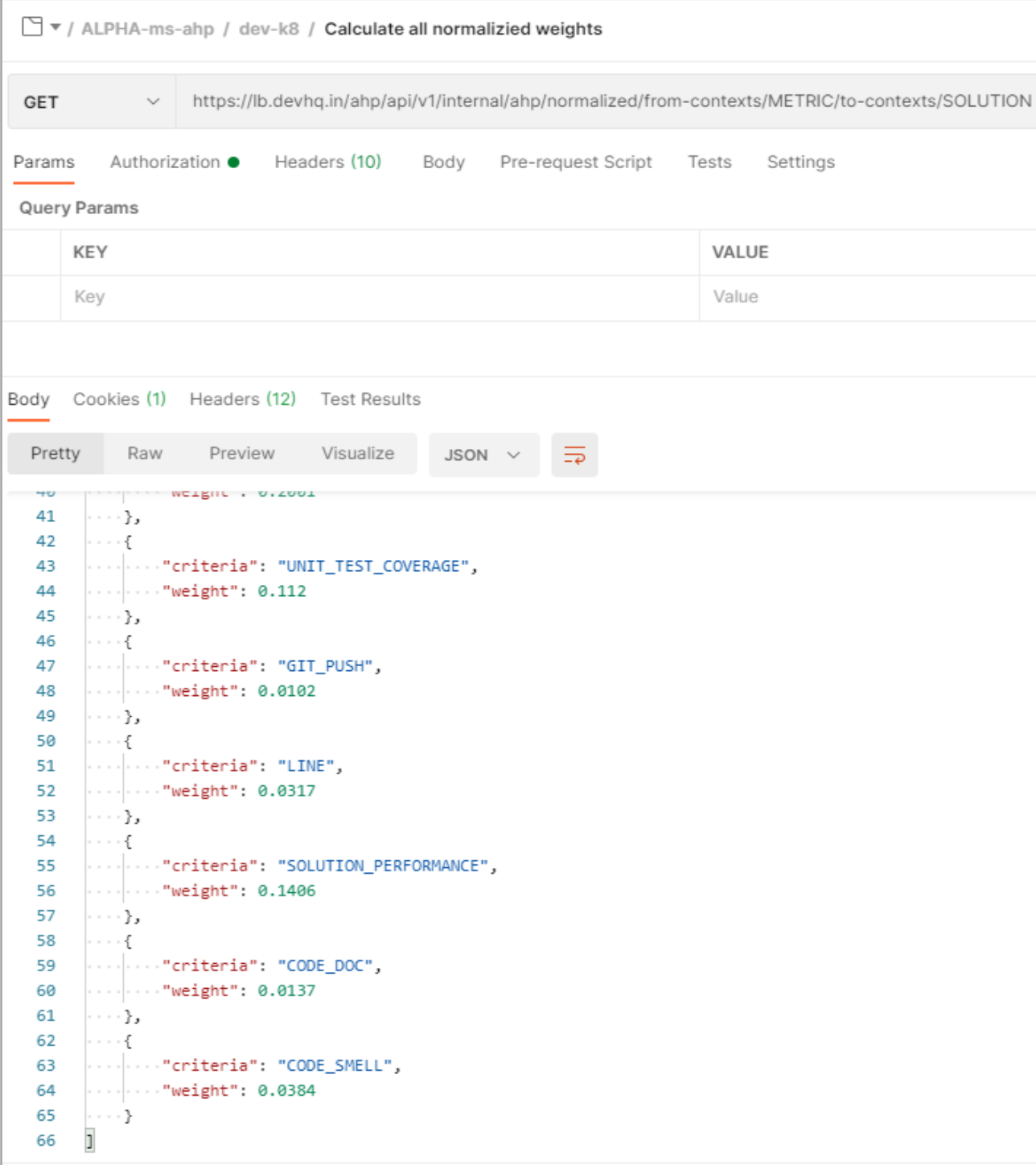

Fig. 5. Getting the weights for each criteria via AHP REST API 
Conclusions. The method of analysis of hierarchies has been applied in the software solution, which could assess technical performance and qualifications of candidates transparently without involving any human in the decision-making process. There is a list of certain technical metrics, which are used in the assessment. These metrics have certain priorities over others. AHP identifies which metric has to be more prioritized over other metrics and how the priorities should be evaluated with numbers.

In conclusion, AHP is used in the following use-cases for the software solution:

- Calculation of priorities of performance metrics on any challenge solution. This means for any challenge we specify all available criteria, hence it calculates the scoring for all existing performance metrics accordingly after the deadline of the challenge.

- Calculation of priorities of performance metrics on topics, skills, and competencies. The priority here is used further to figure out how much scores of performance metrics have an influence on the assessment of users' topics, skills, and competencies on the given challenge.

- Calculation of priorities of topics on skills. It is defined what topics encompass what skills.

Thus, AHP provides a proper solution to define the decision-making system flexibly and transparently.

\section{REFERENCES}

1. Badalov AK, Mehdialiyev AI, "On the establishment of a development scenario of higher education". Collection of scientific articles of "Informatics" Scientific Production Association of the State Committee of Science and Technology of the Republic of Azerbaijan. Baku, 1995.

2. Mehdialiyev AI "Systematic approach to municipal governance problems." Materials of the scientific session on "Sovereignty, democracy, public administration" dedicated to the 10th anniversary of the state independence of Azerbaijan. Baku, 2002.

3. Mehdialiyev AI Abstracts of the international conference "On the international and regional significance of the Baku-Tbilisi-Ceyhan oil pipeline" "On the forecasting model of international oil and gas pipelines". Baku, 2002.

4. Mehdialiyev AI "Mathematical model of substantiation and development forecast of Baku-Tbilisi-Ceyhan oil pipeline". Materials of the international conference "Azerbaijan after independence", Baku, 2003.

5. Mehdialiyev AI Application of expert assessments to municipal administration. "The main directions of sustainable development in modern times", Materials of the Republican Scientific-Practical Conference, p. 68-76. Baku, 2017.

6. Mekhtaliev AI On the solution of the problem of management and forecasting by the method of analysis of hierarchies. International Conference "Dynamic Systems: Sustainability, Management, Optimization" (DSSCO'08). Minsk, 2008.

7. Mehtaliev AI "Mathematical model of distribution of oil revenues in Azerbaijan". VIII International FAM'2009 conference. Program and thesis. Krasnoyarsk, 2009.

8. Saaty Thomas L. The analytic Hierarchy process. New York McGraw-Hill, 1980.

9. Thomas L. Saaty, Kevin P. Kearns. Analytical Planing. The Organizations of Systems, 1985.

10. Fegan Aliyev, Aghamehdi Mehdialiyev, Nurmammad Mammadov and Farhad Aliyev. Development of mathematical model of intellectual building in Baku city. Proceedings of the second international conference "Problems of cybernetics and informatics", PCI'2008, deducated to the 50th Anniversary of the ICT in Azerbaijan, Volume I

11. Mehdialiyev A.I., H.M.Misirov Mathematical model for management of the municipal authorities. Open Access Peer-Reviewed Journal "Science Review" 4(21), May 2019. Warsaw, Poland. p. 3-8. 